\section{RZTRAN: A FORTRAN IV program for weighted averaging of Pearson rs via Fisher's z transformation}

\section{KENN FINSTUEN \\ Our Lady of the Lake University, San Antonio, Texas 78285}

In psychological research, it frequently becomes necessary to compare or combine correlation coefficients from various subsamples. Since the Pearson $r$ is an index number anc is not equally scaled in terms of metric units, direct comparison by inspection or the calculation of a simple arithmetic mean will not suffice for all purposes. The Fisher (1950) logarithmic transformation from $r$ to $z$ solves these problems, since the distribution of Fisher's $\mathrm{z}$ is approximately normal. Transformed rs are directly comparable to zs and allow a weighted average value, $\bar{z}_{r}$, to be computed. The $\bar{z}_{r}$ value, once computed, may then be transformed back to a Pearson $\mathrm{r}$ equivalent (Guilford, 1965, pp. 347-349; McNemar, 1969, pp. 157-158). McNemar (1969, p. 158) also outlines a test of significance for the average weighted $r$, using $\bar{z}_{r}$ and its standard error. Direct averaging of Pearson $r$ values is permissible when $r$ is less than \pm .25 , since $\mathrm{z}$ equals $\mathrm{r}$ within this range to two decimal places (Guilford, 1965, p. 589). However, such an average is not weighted by sample sizes.

In the consideration of which transformed $r$ values to include in a weighted average, two techniques are readily available. The first is based upon the upper and lower limits describing the $99 \%$ interval of statistical confidence around a given $z$. The confidence interval is computed using the standard error of $z$, which is a function of the size of the sample upon which $r$ is based. The confidence limits for $\mathrm{z}$ may then be converted back to correlation values, permitting confidence comparisons of r (Edwards, 1976, pp. 87-89).

Another method in determining if correlation coefficients can be averaged is also suggested by Edwards (1976, pp. 91.92). The technique assesses the homogeneity of the weighted $\mathrm{z}$ values using a chi-square test for independence. If the chi-square value indicates that the rs could have come from the same population, a weighted average of several rs would be a much more reliable estimate of correlation in the population.

Program Description. The RZTRAN program is designed to transform correlation coefficients into $\dot{z}$ values and to compute the standard error of each $z$ along with the lower and upper confidence limits for both $\mathrm{z}$ and its associated $\mathrm{r}$ at the $99 \%$ level. The program then enters the transformed $\mathrm{r}$ coefficients into a weighted average equation and provides both the weighted average, $\bar{z}_{r}$, and its corresponding $r$ equivalent. Next, McNemar's (1969) standard error for $\bar{z}_{\mathrm{r}}$ is calculated. Any significance testing concerning the average $r$ may then be done using $\overline{\mathrm{z}}_{\mathrm{r}}$ and its standard error. Edward's (1976) chi-square test for homogeneity of correlation coefficients is also computed.

Input. The program is designed for batch-mode operation. The user provides the number of correlation coefficients and the title of the study on a control card. The correlation coefficients with associated sample sizes are entered, one set per card, following the control card. RZTRAN also allows an eight-field descriptive sample name.

Output. The output consists of descriptive header information and an $8 \mathrm{x} n$ table comprised of Pearson's $\mathrm{r}$, the Fisher $\mathrm{z}$ transfonnation, the standard error of each $\mathrm{z}$, the sample size, and lower and upper confidence limits for both $\mathrm{z}$ and $\mathrm{r}$ for every $\mathrm{n}$ entry. A summary data table is also included, indicating the number ( $n$ ) of coefficients considered, an intermediate sum for the weighted $\mathrm{zs}$, the aggregated sample size adjusted by degrees of freedom, the weighted average $\bar{z}_{I}$ and $r$ equivalent, McNemar's (1969) standard error of $\bar{z}_{\mathrm{r}}$, and Edward's (1976) chi-square homogeneity test with the associated degrees of freedom. The program also prints a correlation graph upon which $r$ values, their associated $99 \%$ confidence limits, and the weighted average may be plotted.

Limitations. Up to $100 \mathrm{r}$ coefficients and sample size sets may be entered. There may be no missing data. Correlations can be entered in up to five decimal places and may be either positive or negative. The sample size parameters may vary from 2 to 999 . Although the program does not test the significance of differences between rs entered, output data for the $\mathrm{z}$ transformation and the standard error of $z$ greatly simplify such a calculation (cf. Edwards, 1976, pp. 89-90). One note of caution is engendered, however: Since the Fisher $z$ is not a standard score, it should not be treated as such.

Program Specifications. RZTRAN was written for a FORTRAN IV compiler and is currently in use on a UNIVAC 1108 multiprocessor system.

Availability. A listing of RZTRAN, including documentation and a sample run, may be obtained by writing to Kenn Finstuen, Department of Psychology, Our Lady of the Lake University, San Antonio, Texas 78285.

\section{REFERENCES}

Edwards, A. L. An introduction to linear regression and correlation. San Francisco: W. H. Freeman, 1976.

FISHER, R. A. Statistical methods for research workers (11th ed.), New York: Hafner, 1950.

GUILFORD, J. P. Fundamental statistics in psychology and education (4th ed.). McGraw-Hill, 1965.

MCNemar, Q. Psychological statistics (4th ed.). New York: Wiley, 1969.

(Accepted for publication June 29, 1978.) 